\title{
Assessing the temporally dynamic parameters in hydrological models: dynamic operations and evolutionary processes
}

Tian Lan ${ }^{1,4}$, Kairong Lin ${ }^{1,2,3}$, Chong-Yu Xu ${ }^{4}$, Zhiyong Liü ${ }^{2,3}$, Huayang $\mathrm{Cai}^{3}$

${ }^{1}$ Center of Water Resources and Environment Research, Sun Yat-sen University, Guangzhou, 510275, China.

${ }^{2}$ Guangdong Key Laboratory of Oceanic Civil Engineering, Sun Yat-sen University, Guangzhou, 510275, China.

${ }^{3}$ Southern Marine Science and Engineering Guangdong Laboratory (Zhuahai), 519000, China.

${ }^{4}$ Department of Geosciences, University of Oslo, P.O. Box 1047 Blindern, 0316 Oslo, Norway

Correspondence to: Kairong Lin (linkr@mail.sysu.eu.cn) 


\section{Contents of this file}

1 Techniques

1.1 SCE-UA algorithm

1.2 Fitness landscape

1.3 Violin plot

1.4 Parallel coordinates

1.5 Maximal information coefficient (MIC)

1.6 HYMOD model structure

2 Results

References

\section{Introduction}

This supporting information includes two sections that support the analysis. The introduction of the techniques is used to support the Methodology section in the main manuscript. The Results section is used to supplement the Results section in the main manuscript. 


\section{Techniques}

\subsection{SCE-UA algorithm}

The shuffled complex evolution approach (SCE-UA), as an effective global optimization method, is a commonly used algorithm, because it is open source and was the first algorithm aimed specifically at calibrating hydrological models (Khakbaz and Kazeminezhad, 2012; Eckhardt and Arnold, 2001; Duan et al., 1994; Sorooshian et al., 1993). The technical details about the SCEUA can be shown in the flowchart (see Figure S1) (Duan et al., 1994). In the SCE-UA, the upper limit of the objective function evaluation is set to 10,000 times.

The stopping criteria are applied to prevent premature termination, while also avoiding unnecessary computations. There are three stopping criteria in SCE-UA. (1) The maximum number of function evaluations has been reached; this means that the optimization algorithm may not converge at the end of the run. (2) The population has prematurely converged to a pre-specified small geometric range, which indicates that the global convergence has failed to local optima. The method has failed to a zone that it thinks is not amongst the best. It cannot know that it is in a global optimum. (3) The improvement of the best point in the last loops is less than the specified threshold. It indicates that global convergence has been achieved. More details on the SCE-UA are provided in the supporting information (section 1.1). Certain stopping criteria of the global optimization algorithm lead to possible failure in finding the global optimum, which may lead to abnormal or unreasonable optimal parameters. Namely, the optimized parameters cannot effectively characterize the physical systems of the hydrological models (or catchment response behavior).

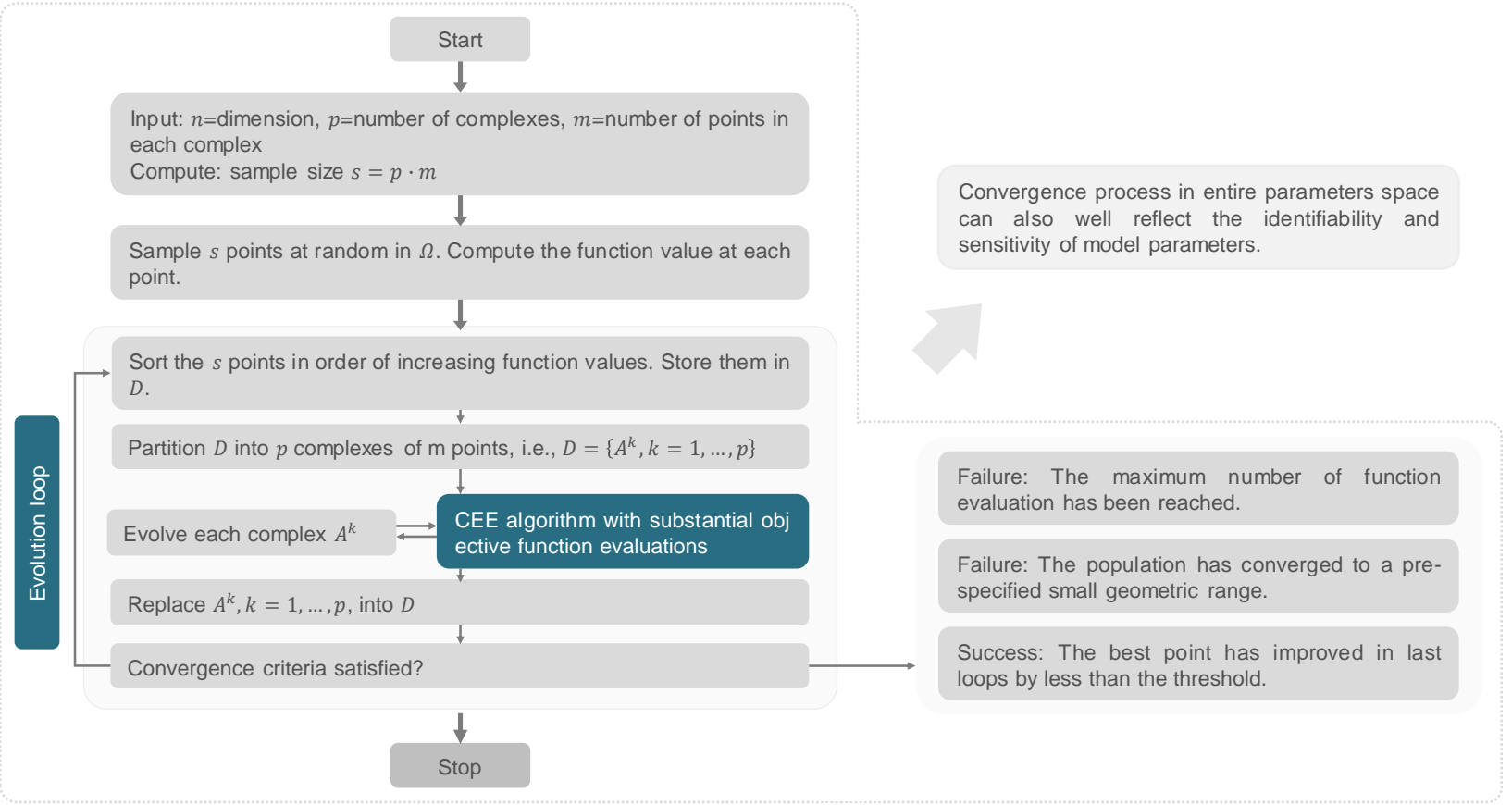

Figure S1. The flowchart of the SCE-UA algorithm (Duan et al., 1992, 1993, 1994).

\subsection{Fitness landscape}

A potent metaphor in global optimization is the fitness landscape (Aldrich, 1997). In evolutionary biology, fitness landscapes have been developed to visualize the relationship between the genotypes or phenotypes in a given population and their corresponding reproduction probability (Wright, 1932; Kauffman, 1993; Dawkins, 1997; Gavrilets, 2004). The idea of such visualizations goes 
back to Wright (1932), who used level contours diagrams in order to outline the effects of selection, mutation, and crossover on the capabilities of populations to escape local optimal configurations. The evolutionary algorithm research community has widely adopted the fitness landscapes as a relation between individuals and their objective values (Mitchell, 1998).

\subsection{Violin plot}

A violin plot is a combination of a Box Plot and a Density Plot showing more details of data distribution. As shown in Figure S2, the thick black bar in the center represents the interquartile range. The white dot represents the median. The thin black line is extended from the thick black bar and represents the 95\% confidence intervals. On each side of the thin black line is a kernel density estimation to show the distribution shape of the data. Wider sections of the violin plot represent a higher probability that members of the population will take on the given value; the skinnier sections represent a lower probability (Hintze and Nelson, 1998). The violin plots can exactly show the kernel density distribution, avoiding the overlapping traditional density plot occur to become difficult to identify. Moreover, unlike bar graphs with means and error bars, violin plots contain all data points, which makes them an excellent tool to visualize samples of small sizes. Violin plots are perfectly appropriate even if the data do not conform to normal distribution. They work well to visualize both quantitative and qualitative data.

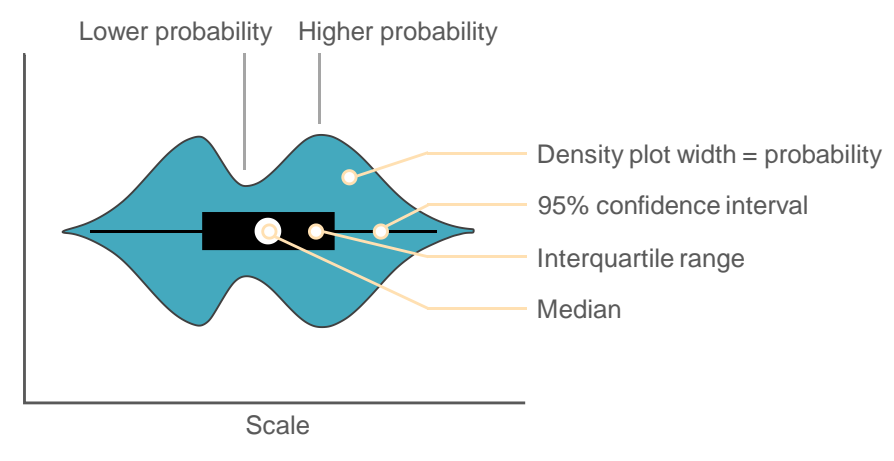

Figure S2. Anatomy of a violin plot.

\subsection{Parallel coordinates}

The theory of parallel coordinates has been developed rigorously, and its point-line duality has been successively generalized to higher dimensions (Heer et al., 2010; Shenghui and Mueller, 2015). In order to show a set of points in an $n$-dimensional space, a backdrop is drawn consisting of $n$ parallel lines, typically vertical and equally spaced. That is, a point in $n$-dimensional space is represented as a polyline with vertices on the parallel axes. Each axis corresponds to a variable, and each data item, having values for all variables, is represented as a series of line segments intersecting the axes at the corresponding values (Zhou and Weiskopf, 2018). An excellent complication of current research on parallel coordinates can be found in the state-of-the-art report by Heinrich and Weiskopf (2013).

\subsection{Maximal information coefficient (MIC)}

The MIC was proposed by Reshe et al. (2011) and did not rely on the distributional assumptions of the datasets. This measurement approach captures extensive mutual information of the variables for functional and non-functional relationships. For functional relationships, the MIC algorithm provides a score that is roughly equivalent to the coefficient of determination $\left(R^{2}\right)$ of the datasets. However, its statistical power reduced in detecting the associations in setting with a low sample size (Heller et al., 2012). 


\subsection{HYMOD model structure}

For illustration purposes, the HYMOD model (Moore, 1985; Wagener et al., 2001; Vrugt et al., 2002; Yadav et al., 2007; de Vos et al., 2010; Pathiraja et al., 2018), with a simple and commonly used lumped model structure, is utilized. The HYMOD model consists of a simple rainfall excess model based on the probability-distributed moisture store, which characterizes the catchment storage as a Pareto distribution of buckets of varying depth as the soil moisture accounting component. It routes through three parallel tanks for quick flow and a tank for slow flow and required five adjustable parameters: $H_{U Z}, B, \alpha, K_{q}$ and $K_{S} . X H_{U Z}$ and $X C_{U Z}$ are state variables characterizing the upper soil moisture content; $A E$ is actual evapotranspiration which is calculated by linear correlations between the soil moisture state and the potential evapotranspiration; effP is effective precipitation; $O \mathrm{~V}$ is excess precipitation to routing module generated from overflow of soil moisture accounting component; See Moore (1985) for a detailed description of the soil moisture accounting model; $X_{q 1}, X_{q 2}, X_{q 3}$ and $X_{s}$ are the state variables of the individual tanks of the routing module; $Q_{q}$ and $Q_{s}$ are the flow values generated from the quick- and slow-flow tanks, respectively.

\section{Results}
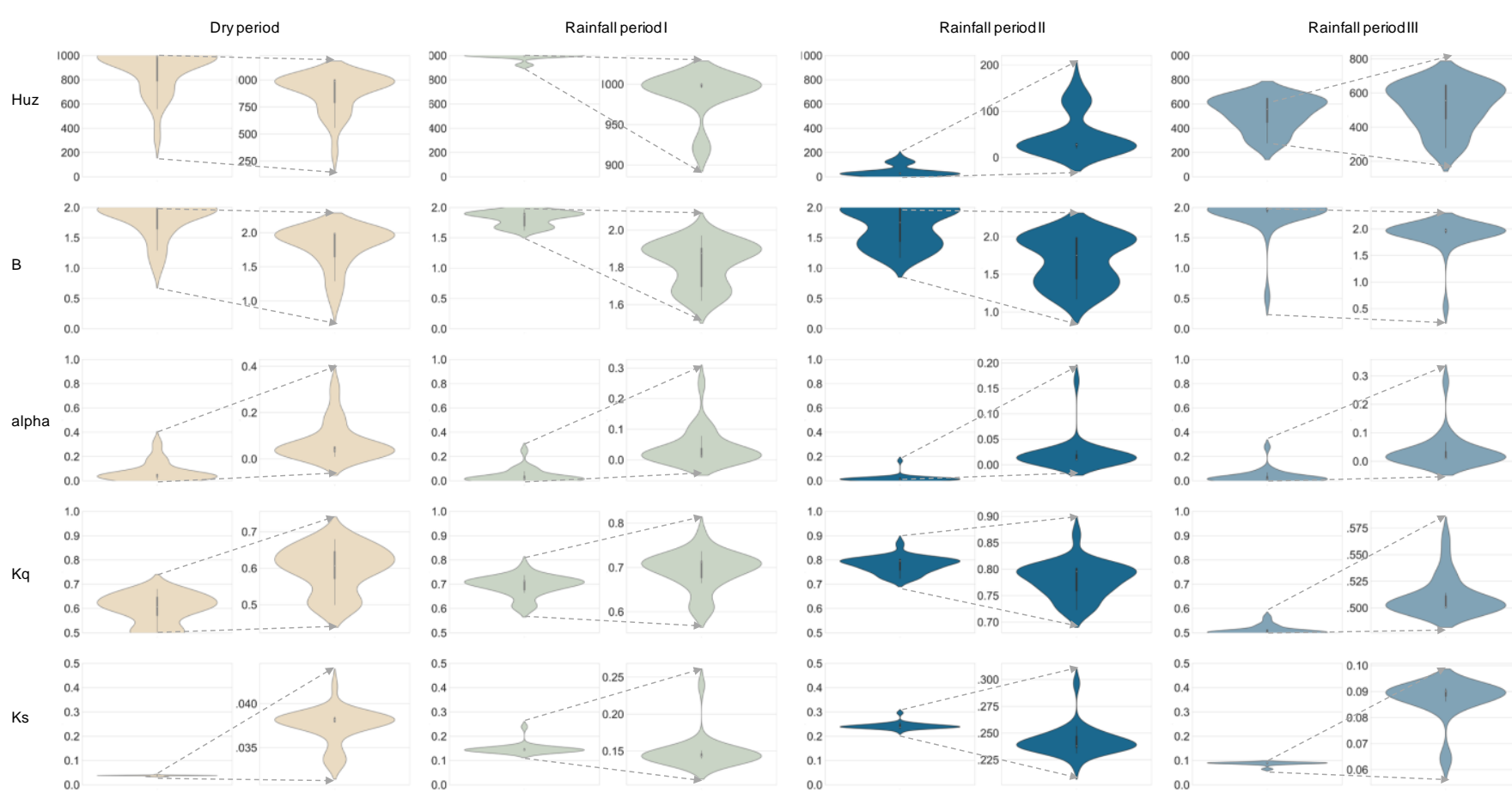

Figure S3. Evolutionary processes of dynamic parameters in the individual parameter spaces in the Mumahe basin. 

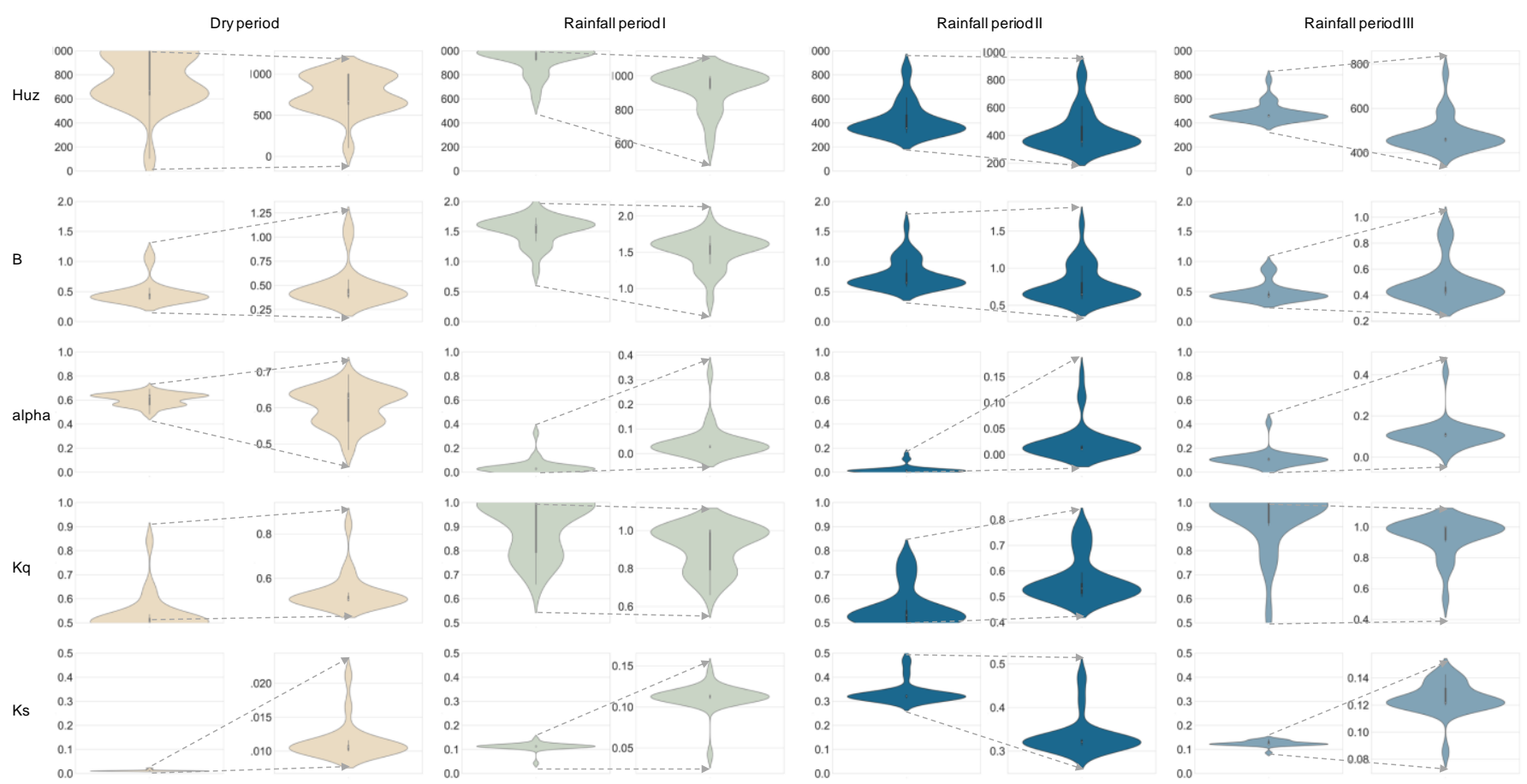

Figure S4. Evolutionary processes of dynamic parameters in the individual parameter spaces in the Xunhe basin. 

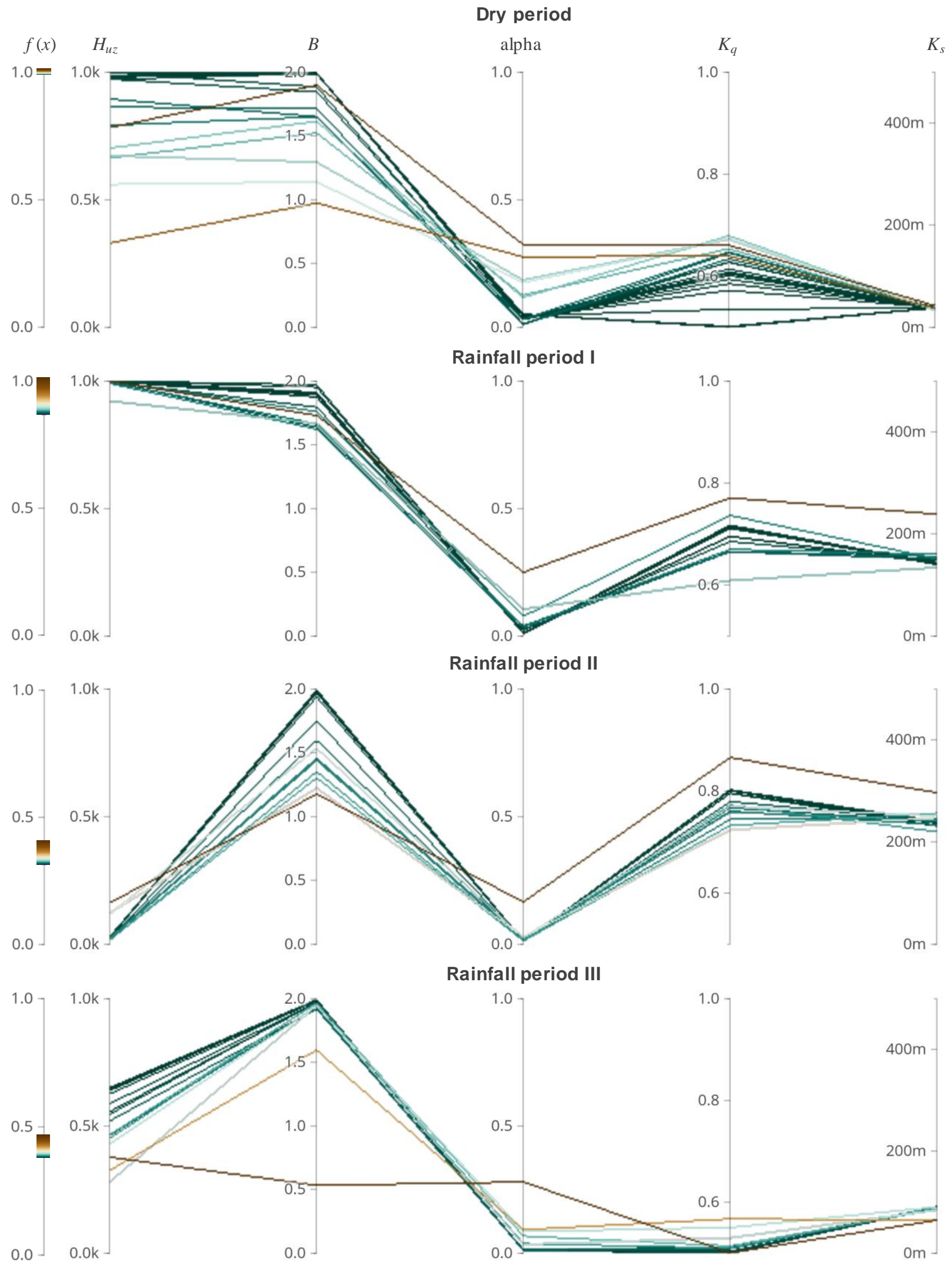

Figure S5. Evolutionary processes of dynamic parameters in multi-parameter space in the Mumahe basin. 


\section{Dry period}
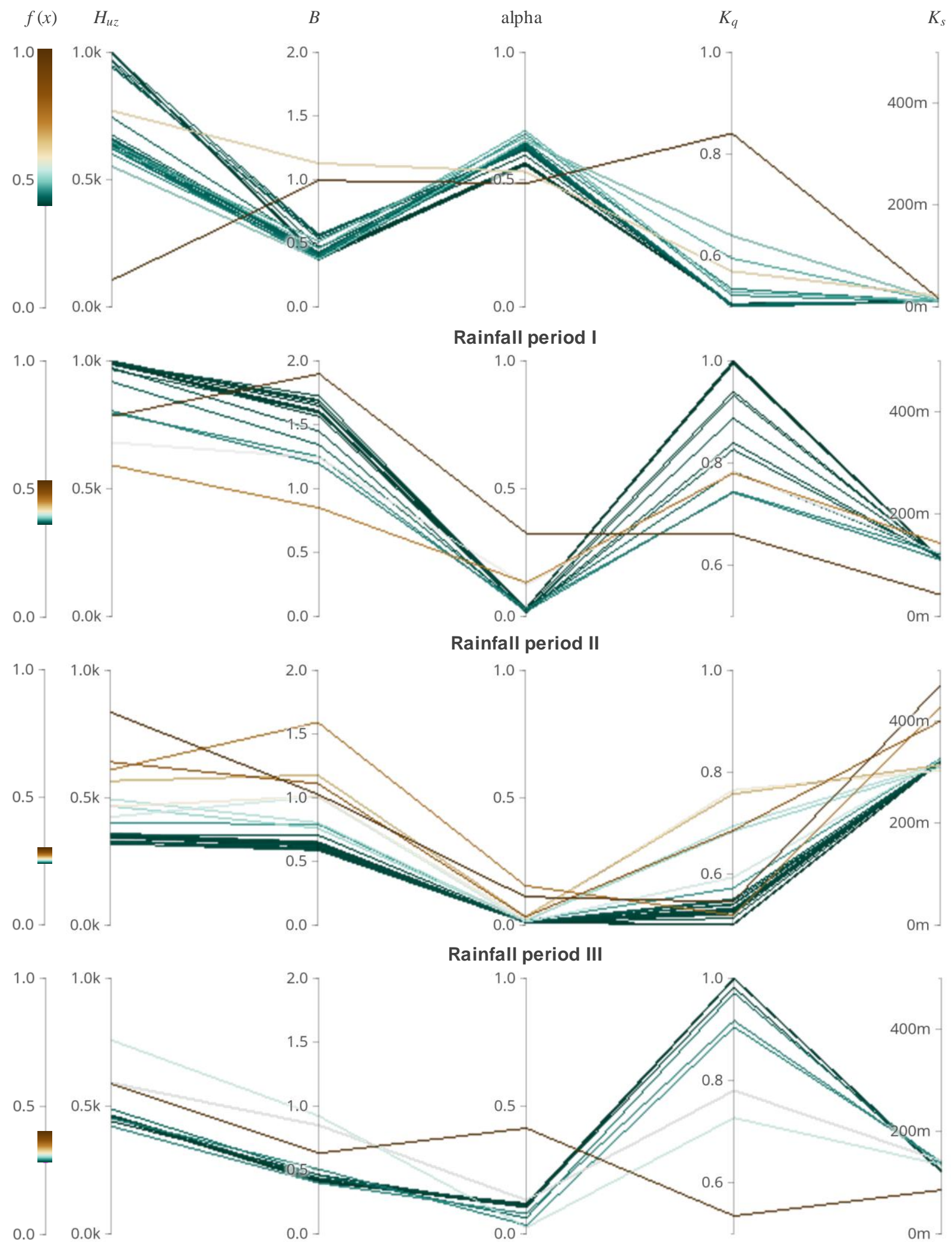

Figure S6. Evolutionary processes of dynamic parameters in multi-parameter space in the Xunhe basin. 
Dry period
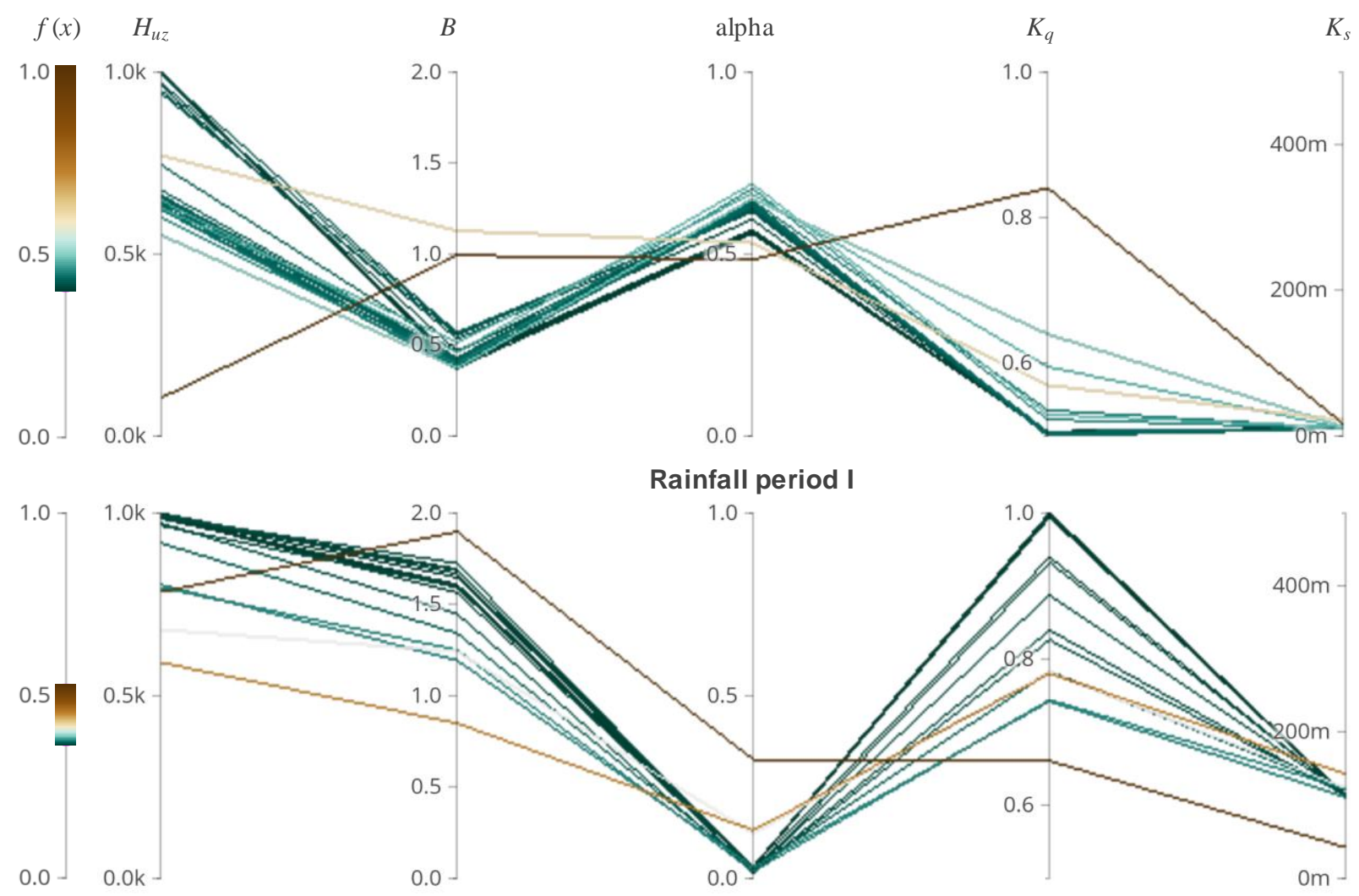

Rainfall period II

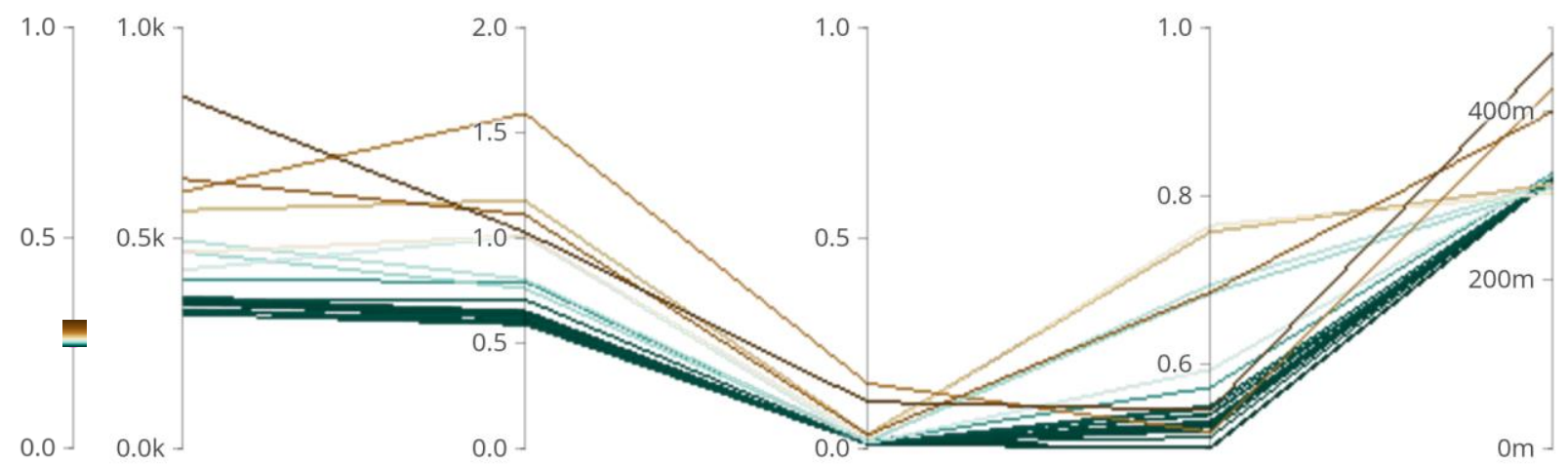

Rainfall period III

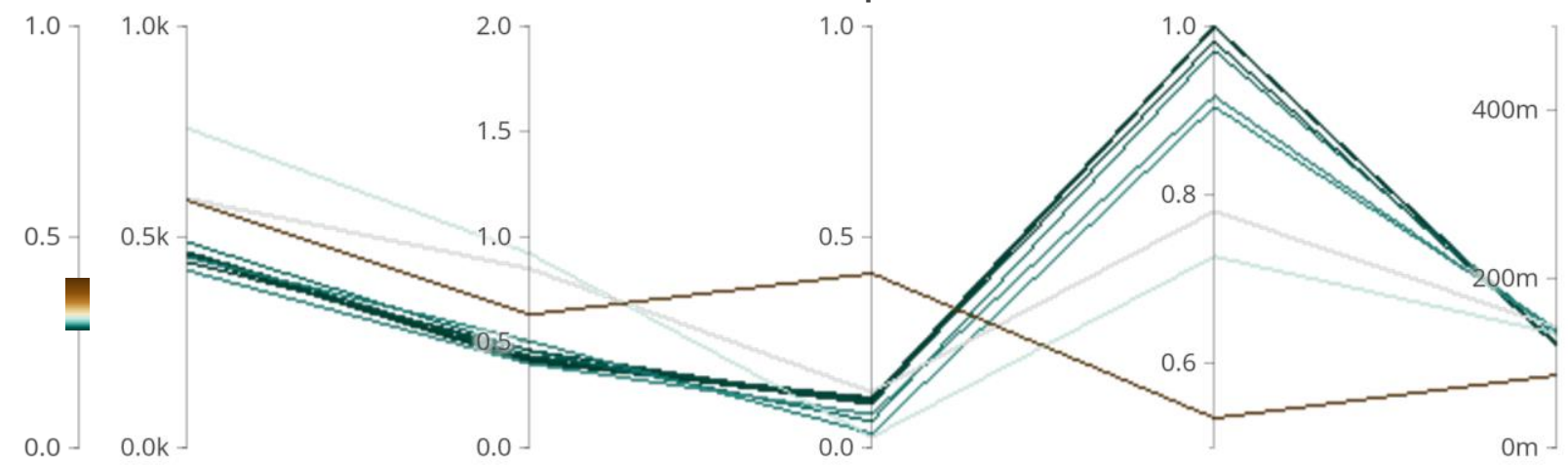

Figure S7. Evolutionary processes of dynamic parameters with magnified details on the axes in multi-parameter space in the Mumahe basin. 


\section{Dry period}

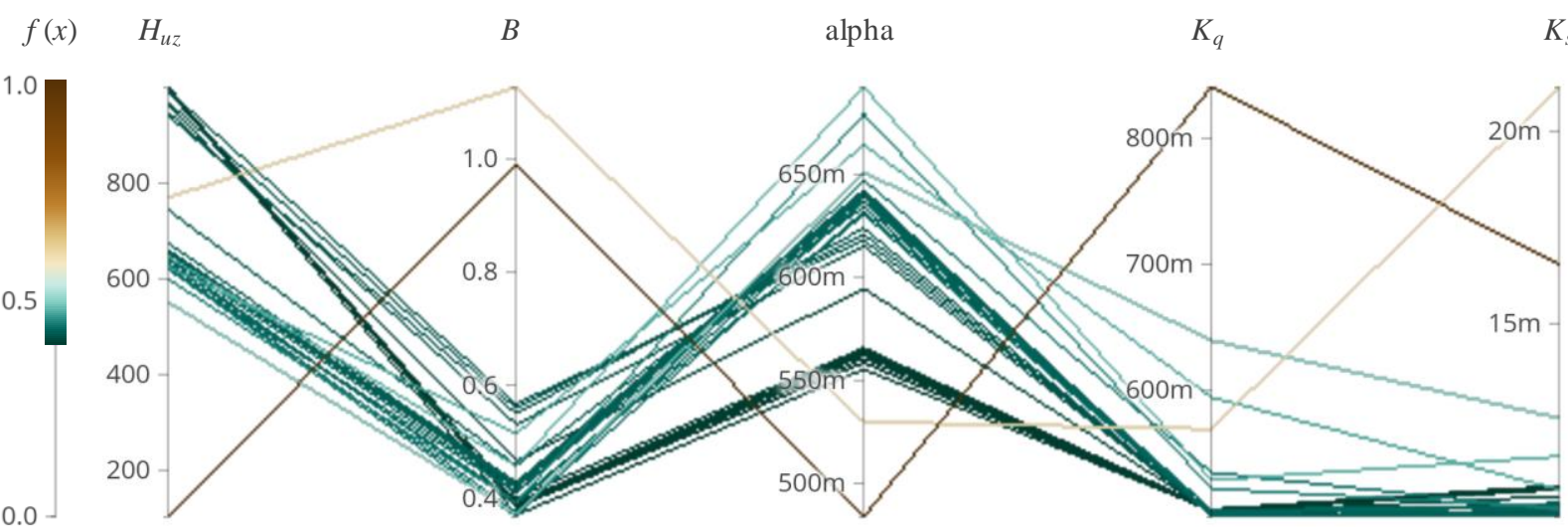

Rainfall period I

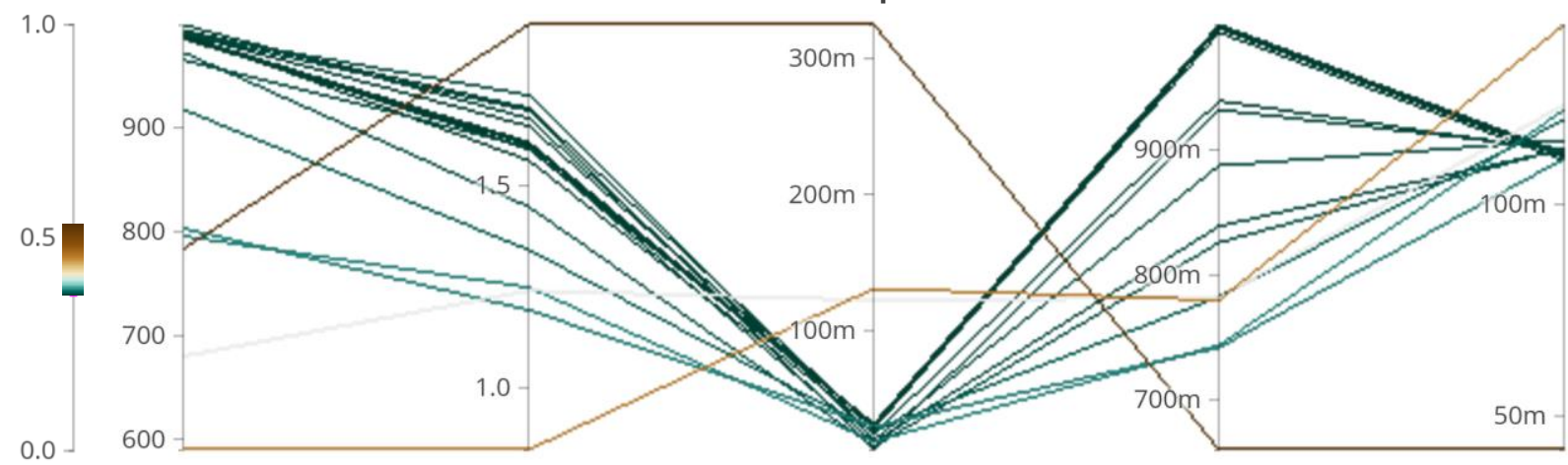

Rainfall period II

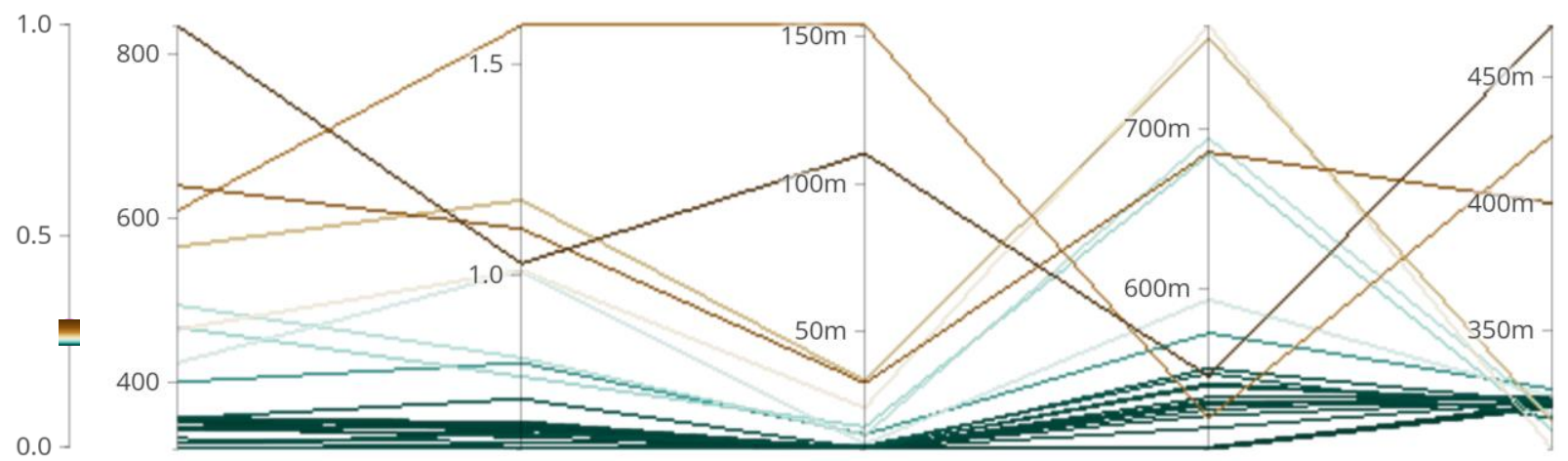

Rainfall period III

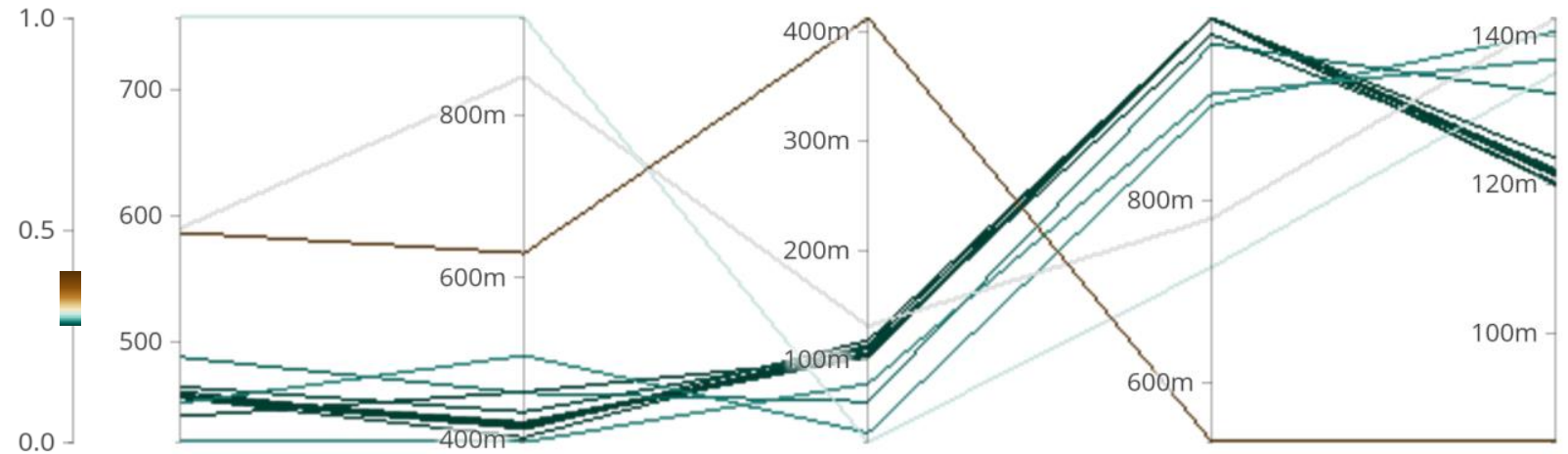

Figure S8. Evolutionary processes of dynamic parameters with magnified details on the axes in multi-parameter space in the Xunhe basin. 


\section{References}

Aldrich, J.: R.A. Fisher and the making of maximum likelihood 1912-1922, Statist. Sci., 12, 162-176, 10.1214/ss/1030037906, 1997.

Dawkins, R.: Climbing mount improbable, WW Norton \& Company, 1997.

de Vos, N. J., Rientjes, T. H. M., and Gupta, H. V.: Diagnostic evaluation of conceptual rainfall-runoff models using temporal clustering, Hydrol Process, 24, 2840-2850, 10.1002/hyp.7698, 2010.

Duan, Q., Sorooshian, S., and Gupta, V.: Effective and efficient global optimization for conceptual rainfall-runoff models, Water Resources Research, 28, 1015-1031, 10.1029/91WR02985, 1992.

Duan, Q., Sorooshian, S., and Gupta, V. K.: Optimal use of the SCE-UA global optimization method for calibrating watershed models, Journal of hydrology, 158, 265-284, 1994.

Duan, Q. Y., Gupta, V. K., and Sorooshian, S.: Shuffled Complex Evolution Approach for Effective and Efficient Global Minimization, Journal of Optimization Theory and Applications, 76, 501-521, Doi 10.1007/Bf00939380, 1993.

Eckhardt, K., and Arnold, J. G.: Automatic calibration of a distributed catchment model, J Hydrol, 251, 103-109, Doi 10.1016/S0022-1694(01)00429-2, 2001.

Gavrilets, S.: Fitness landscapes and the origin of species (MPB-41), Princeton University Press, 2004.

Heer, J., Bostock, M., and Ogievetsky, V.: A tour through the visualization zoo, Commun. ACM, 53, 59-67, 10.1145/1743546.1743567, 2010.

Heinrich, J., and Weiskopf, D.: State of the Art of Parallel Coordinates, Eurographics, 2013,

Heller, R., Heller, Y., and Gorfine, M.: A consistent multivariate test of association based on ranks of distances, Biometrika, 100, 503-510, 10.1093/biomet/ass070, 2012.

Hintze, J. L., and Nelson, R. D.: Violin plots: a box plot-density trace synergism, The American Statistician, 52, 181-184, https://doi.org/10.2307/2685478, 1998.

Kauffman, S. A.: The origins of order: Self-organization and selection in evolution, OUP USA, 1993.

Khakbaz, F., and Kazeminezhad, M.: Work hardening and mechanical properties of severely deformed AA3003 by constrained groove pressing, Journal of Manufacturing Processes, 14, 20-25, 10.1016/j.jmapro.2011.07.001, 2012.

Mitchell, M.: An introduction to genetic algorithms, MIT press, 1998.

Moore, R. J.: The probability-distributed principle and runoff production at point and basin scales, Hydrological Sciences Journal, 30, 273-297, 10.1080/02626668509490989, 1985.

Pathiraja, S., Anghileri, D., Burlando, P., Sharma, A., Marshall, L., and Moradkhani, H.: Time-varying parameter models for catchments with land use change: the importance of model structure, Hydrol Earth Syst Sc, 22, 2903-2919, 10.5194/hess-222903-2018, 2018.

Reshe, D. N., Reshef, Y. A., Finucane, H. K., Grossman, S. R., McVean, G., Turnbaugh, P. J., Lander, E. S., Mitzenmacher, M., and Sabeti, P. C.: Detecting novel associations in large data sets, Science, 334, 1518-1524, 
https://doi.org/10.1126/science.1205438, 2011.

Shenghui, C., and Mueller, K.: Improving the fidelity of contextual data layouts using a Generalized Barycentric Coordinates framework, 2015 IEEE Pacific Visualization Symposium (PacificVis), 2015, 295-302.

Sorooshian, S., Duan, Q., and Gupta, V. K.: Calibration of rainfall-runoff models: Application of global optimization to the Sacramento Soil Moisture Accounting Model, Water Resour. Res., 29, 1185-1194, https://doi.org/10.1029/92wr02617, 1993.

Vrugt, J. A., Bouten, W., Gupta, H. V., and Sorooshian, S.: Toward improved identifiability of hydrologic model parameters: The information content of experimental data, Water Resources Research, 38, 48-41-48-13, doi:10.1029/2001WR001118, 2002.

Wagener, T., Boyle, D. P., Lees, M. J., Wheater, H. S., Gupta, H. V., and Sorooshian, S.: A framework for development and application of hydrological models, Hydrol. Earth Syst. Sci., 5, 13-26, 10.5194/hess-5-13-2001, 2001.

Wright, S.: The roles of mutation, inbreeding, crossbreeding, and selection in evolution, na, 1932.

Yadav, M., Wagener, T., and Gupta, H.: Regionalization of constraints on expected watershed response behavior for improved predictions in ungauged basins, Advances in Water Resources, 30, 1756-1774, https://doi.org/10.1016/j.advwatres.2007.01.005, 2007.

Zhou, L., and Weiskopf, D.: Indexed-Points Parallel Coordinates Visualization of Multivariate Correlations, IEEE Trans Vis Comput Graph, 24, 1997-2010, 10.1109/TVCG.2017.2698041, 2018. 